\title{
Stable recombination hotspots in birds
}

\section{Authors}

Sonal Singhal ${ }^{\star 1,2+}$, Ellen M. Leffler ${ }^{\star 3,4}$, Keerthi Sannareddy ${ }^{3}$, Isaac Turner ${ }^{4}$, Oliver Venn ${ }^{4}$, Daniel M. Hooper ${ }^{5}$, Alva I. Strand ${ }^{1}$, Qiye Li ${ }^{6}$, Brian Raney ${ }^{7}$, Christopher N. Balakrishnan ${ }^{8}$, Simon C. Griffith $^{9}$, Gil McVean ${ }^{4}$, Molly Przeworski ${ }^{1,2+}$

\author{
Affiliations \\ ${ }^{1}$ Columbia University, Dept. of Biological Sciences, New York, NY \\ ${ }^{2}$ Columbia University, Dept. of Systems Biology, New York, NY \\ ${ }^{3}$ University of Chicago, Dept. of Human Genetics, Chicago, IL \\ ${ }^{4}$ University of Oxford, Wellcome Trust Centre for Human Genetics, Oxford, United Kingdom \\ ${ }^{5}$ Committee on Evolutionary Biology, University of Chicago, Chicago, IL \\ ${ }^{6}$ China National GeneBank, BGI-Shenzhen, China \\ ${ }^{7}$ University of California Santa Cruz, Center for Biomolecular Science \& Engineering, Santa \\ Cruz, CA \\ ${ }^{8}$ East Carolina University, Biology, Greenville, NC \\ ${ }^{9}$ Macquarie University, Dept. of Biological Sciences, Sydney, Australia \\ * co-first authors \\ ${ }^{+}$to whom correspondence should be addressed
}

\section{Abstract}

Although the DNA-binding protein PRDM9 plays a critical role in the specification of meiotic recombination hotspots in mice and apes, it appears to be absent from many vertebrate species, including birds. To learn about the determinants of fine-scale recombination rates and their evolution in natural populations lacking PRDM9, we inferred fine-scale recombination maps from population resequencing data for two bird species, the zebra finch Taeniopygia guttata, and the long-tailed finch, Poephila acuticauda, whose divergence is on par with that between human and chimpanzee. We find that both bird species have hotspots, and these are enriched near CpG islands and transcription start sites. In sharp contrast to what is seen in mice and apes, the hotspots are largely shared between the two species, with indirect evidence of conservation extending across bird species tens of millions of years diverged. These observations link the evolution of hotspots to their genetic architecture, suggesting that in the absence of PRDM9 binding specificity, accessibility of the genome to the cellular recombination machinery, particularly around functional genomic elements, both enables increased recombination and constrains its evolution.

\section{One-Sentence Summary}

We show the fine-scale recombination landscape is stable across tens of millions of years in birds, in sharp contrast to what is seen in primates and mice. 


\section{Main Text}

Meiotic recombination is a ubiquitous and fundamental genetic process that shapes variation in populations, yet our understanding of its underlying mechanisms is based only on a handful of model organisms, scattered throughout the tree of life. One pattern shared among most sexually reproducing species is that meiotic recombination tends to occur in short segments of 100 s to 1000 s of base pairs, termed "recombination hotspots" (1). In apes and mice, the location of hotspots is largely determined by PRDM9, a zinc-finger protein that binds to specific motifs in the genome during meiotic prophase and generates H3K4me3 marks, eventually leading to double-strand breaks (DSBs) and both crossover and non-crossover resolutions (2-5). The zinc-finger domain of PRDM9 evolves quickly, with evidence of positive selection on residues in contact with DNA $(2,6)$, and as a result, there is rapid turnover of hotspot locations across species, subspecies, and populations (7-9).

Whereas $P R D M 9$ plays a pivotal role in controlling recombination localization in mice and apes, many species lacking PRDM9 nonetheless have hotspots (6). An artificial example is provided by mice knockouts for PRDM9. Although sterile, they make similar numbers of DSBs as wild-type mice, and their recombination hotspots appear to default to residual H3K4me3 mark locations, notably at promoters (10). A natural but puzzling example is provided by canids, who carry premature stop codons in PRDM9, yet are able to recombine and remain fertile (11). Like mouse PRDM9 knockouts, in canids and in other species without PRDM9 such as Saccharomyces cerevisae and Arabidopsis thaliana, hotspots tend to occur at promoters or other regions with promoter-like features (11-13). In some taxa without PRDM9, notably Drosophila species (14, 15), honeybees (16), and Caenorhabditis elegans (17), short, intense recombination hotspots appear to be absent.

To further explore how the absence of PRDM9 shapes the fine-scale recombination landscape and impacts its evolution, we turn to birds, because they have compact and largely syntenic genomes that facilitate comparative study (18) and because an analysis of the chicken genome suggested that birds may not have PRDM9 (6). We first confirmed the absence of PRDM9 in birds by querying the genomes of 48 species of birds, three species of crocodilians, two species of turtles, and one species of lizard for $\operatorname{PRDM9}(19,20)$. We found that only turtles contain putative orthologs with all three PRDM9 domains (Fig. S1). We also found no expression of any PRDM9-like transcripts in RNAseq data from testes tissue of the zebra finch (Taeniopygia guttata). Given the likely absence of $P R D M 9$ in birds, we ask: is recombination nonetheless concentrated in hotspots in these species? If so, how quickly do the hotspots evolve? Where does recombination tend to occur in the genome? To address these questions, we generated whole-genome resequencing data for wild populations from two bird species and inferred fine-scale genetic maps from patterns of linkage disequilibrium. While this approach only generates a historical, sex-averaged map of recombination (21), other methods such as sperm typing, double strand break sequencing, or analyses of pedigrees are impractical for most non-model species (22).

\section{Inferring fine-scale recombination maps}

We generated whole genome resequencing data from wild populations of three species of finch in the family Estrildidae: zebra finch (Taeniopygia guttata; $n=19$ wild, unrelated birds and $n=5$ from a domesticated, nuclear family), long-tailed finch (Poephila acuticauda; $n=20$, including 10 
of each of two, similar subspecies with average autosomal $F_{S T}=0.039$ ), and double-barred finch (Taeniopygia bichenovii; $\mathrm{n}=1$ ) (Fig. 1, Table S1; (23)). Despite extensive incomplete lineage sorting between the species, there is no evidence for gene flow amongst them (Fig. S2). Moreover, nucleotide divergence among the three finch species is similar to that of human, chimpanzee and gorilla, providing a well-matched comparison to apes (8, 9) (Fig. 1).

We mapped reads from all individuals to the zebra finch reference genome $(1 \mathrm{~Gb}$ assembled across 34 chromosomes; (24)) and generated de novo SNP calls for all three species. After filtering for quality, we identified 44.6 million SNPs in zebra finch, 26.2 million SNPs in long-tailed finch, and 3.0 million SNPs in double-barred finch (Table S2). These SNP numbers correspond to autosomal nucleotide diversity of $\pi=0.82 \%$ and $\theta_{\mathrm{w}}=1.37 \%$ in zebra finch and $\pi=0.55 \%$ and $\theta_{\mathrm{w}}=0.73 \%$ in long-tailed finch $(25,26)$, approximately ten times higher than estimates from ape species (27). Assuming a mutation rate per base pair per generation of $7 \mathrm{x}$ $10^{-10}$ (23), this suggests a long-term effective population size $\left(N_{e}\right)$ of $4.8 \times 10^{6}$ and $2.5 \times 10^{6}$ in the zebra finch and long-tailed finch, respectively. Thus, these two species have much larger $N_{e}$ than most other species for which there exist fine-scale recombination maps, with $N_{e}$ more reflective of biodiversity at large (Fig. S3).

Next, we inferred haplotypes for zebra finch and long-tailed finch using a linkagedisequilibrium approach that incorporated phase-informative reads and family phasing $(28,29)$. From the haplotypes, we estimated fine-scale recombination maps using LDhelmet, an approach that has been shown to perform well for species with higher nucleotide diversity (14). The resulting maps estimated genome-wide median recombination rates in zebra finch and in long-tailed finch as $\rho=26.2 / \mathrm{kb}$ and $14.0 / \mathrm{kb}$, respectively, corresponding to a median of 0.14 $\mathrm{cM} / \mathrm{Mb}$ for both species (23). Simulations indicated that we had limited power to identify hotspots at either high recombination rates (Fig. S4), so we restricted our analyses to the 18 largest chromosomes in the zebra finch genome (930 Mb; $91 \%$ of sequence), ignoring microchromosomes in particular. For these 18 chromosomes, our results accord well with recombination maps inferred from a relatively small pedigree-based study of zebra finch (30), with a correlation of 0.91 for rates estimated at the $5 \mathrm{Mb}$ scale (Fig. S5), providing confidence in our inference.

\section{Hotspots and their evolution}

Most of the recombination events in zebra finch and long-tailed finch occur in a narrow portion of the genome, with $82 \%$ and $70 \%$ of events localized to $20 \%$ of the genome in zebra finch and long-tailed finch, respectively (Fig. S6). Given this evidence for heterogeneous recombination, we sought to identify hotspots in the genome. We operationally define hotspots as regions that are at least $2 \mathrm{~kb}$ in length, have at least 5 -fold the background recombination rate estimated for surrounding $80 \mathrm{~kb}$, and are statistically supported as hotspots by a likelihood ratio test (31). This approach yielded 3949 hotspots in zebra finch and 4933 hotspots in longtailed finch (Fig. 2, S7, S8), with one hotspot detected on average every 215 and $179 \mathrm{~kb}$ in the two species, respectively. This difference in hotspot density between species is consistent with simulations that indicate less reliable detection of hotspots when the background population recombination rate is higher, as it is in zebra finch (Fig. S4, Fig. S9). Importantly, the hotspots were detected after aggressively filtering our SNP datasets and show no evidence of having higher phasing error rates than the rest of the genome (Table S4, S5, Fig. S10). 
Considering hotspots as shared if their midpoints occurred within $3 \mathrm{~kb}$ of each other, $73 \%$ of zebra finch hotspots (2874 of 3949 hotspots) were detected as shared between the two species (Fig. S11) when only 3.6\% were expected to overlap by chance (Fig. S12); similar results were obtained under different criteria for hotspot sharing (Table S3). The true fraction of shared hotspots between zebra finch and long-tailed finch is likely higher than observed, because we do not have complete power (Fig. S4) and simulations suggest that we are unlikely to detect spurious cases of hotspot sharing (23). On the other hand, the observed levels of sharing are somewhat lower than expected compared to a model in which all hotspots are identical in the two species (Fig. S13).

Such high levels of hotspot sharing contrasts sharply to results from comparative systems in apes and mice, which exhibit no evidence for hotspot sharing even across populations or species with modest levels of genetic differentiation $(9,10,32)$. As an illustration, when we apply the same criterion as above to humans and chimpanzees, only $10.5 \%$ of hotspots overlap when $7.2 \%$ are expected by chance (Fig. S12). Interestingly, our findings in birds echo those obtained from four species of Saccharomyces yeast, which show nearly complete conservation of hotspot locations and heats across species 15 myr diverged (33). Almost all hotspots in Saccharomyces yeast occur at promoters, which are evolutionarily stable, suggesting that how hotspot locations are specified influences how they evolve (34).

To provide further support for the validity of the inferred hotspots, we tested if they show evidence for GC-biased gene conversion ( $\mathrm{gBGC}$ ), measured as higher equilibrium levels of GC content $\left(\operatorname{GC}^{*}(35,36)\right)$. Because evidence for $\mathrm{gBGC}$ in birds is somewhat indirect $(37,38)$, we first looked for support for gBGC at broad genomic scales, finding a positive relationship between recombination rate and $\mathrm{GC}^{*}$ (Fig. 3A-B). Narrowing our focus to the regions surrounding hotspots, we observe that hotspots exhibit peaked $\mathrm{GC}^{*}$ relative to both flanking sequence and coldspots matched for the same overall GC and CpG content (Fig. 4A-B). A similar phenomenon is seen in intra-species variation data: at hotspots but not matched coldspots, derived alleles segregate at a higher frequency at AT to GC polymorphisms than at GC to AT polymorphisms (Fig. S14). Thus, two independent signatures of recombinationpatterns of linkage disequilibrium and of base composition-converge in demonstrating that finches have recombination hotspots and that these are conserved over much larger time scales than in apes and mice $(8,9,39)$.

After observing the pattern of gBGC at hotspots in zebra finch and long-tailed finch, we tested how far conservation of hotspot locations extends across the avian phylogeny by considering three additional species: double-barred finch (an estimated $~ 3.5$ million years [myr] from zebra finch (23)), for which we had collected genomic data, as well as medium ground finch Geospiza fortis ( 15.5 myr from zebra finch), and collared flycatcher Ficedula albicollis ( 19.1 myr from zebra finch), for which draft assemblies are available $(40,41)$. Because we only had a single diploid genome from these species, we tested for hotspot conservation indirectly by determining if these species had peaks in $\mathrm{GC}^{*}$ at the hotspot locations inferred as shared between zebra finch and long-tailed finch. We find localized $\mathrm{GC}^{*}$ peaks at hotspots in all three species (Fig. 4C-E), suggesting that the conservation of hotspots extends across tens of millions of years of evolution in birds. 
Analyzing fine-scale rates across the genome

Hotspots in zebra finch and long-tailed finch are enriched near transcription start sites (TSSs), with $\sim 18 \%$ occurring within $3 \mathrm{~kb}$ of a TSS (Fig. S15), and near CpG islands (CGIs), with $\sim 40 \%$ of hotspots within $3 \mathrm{~kb}$ of a CGl in both species. Consistently, recombination is nearly two-fold higher near TSSs (Fig. 5A-B), and more than three-fold higher near CGls (Fig. 5C-D). This positive association between proximity to the TSS and recombination rate has been previously reported in a number of species without $P R D M 9$, including $S$. cerevisiae, the monkey-flower Mimulus guttatus, and $A$. thaliana $(12,13,42)$. In turn, the link between CGIs and recombination rates has been found both in species without PRDM9 (dogs; (11), $A$. thaliana; (43)) and, albeit more weakly, in species with PRDM9 (humans and chimpanzees; (9)). Moreover, although the genic recombination rate is positively correlated with expression levels as measured in the testes in the zebra finch (Spearman's $r=0.09, p=2.73 \times 10^{-18}$, Fig. $S 16$ ), the relationship between CGIs and recombination rate remains significant after controlling for expression levels (Spearman's $r=-0.11 ; p=4.32 \times 10^{-27}$ ). This increase in recombination rates near TSSs and CGls supports a model in which, particularly in the absence of PRDM9 binding specificity, recombination is concentrated at functional elements that are accessible to the recombination machinery. Indeed, both TSSs and CGIs have been shown to serve as sites of transcription initiation (44) and to coincide with destabilization of nearby nucleosome occupancy (45).

Under a model in which the recombination machinery tends to target accessible genomic elements, we would not necessarily expect to see enrichment of specific binding motifs associated with hotspot activity. Accordingly then, when we test for motifs enriched in hotspots relative to coldspots, the top motifs in both species are a string of As, which are also enriched in $A$. thaliana and yeast hotspots and which may be nucleosome depleted or facilitate nucleosome removal $(12,46)$, and a number of additional motifs that appear to be reflective of CGls (Fig. S17).

At even finer resolution, recombination rates are higher in exonic than intronic regions (Fig. 5E-F), which has also been seen in A. thaliana (13), dogs (11), and monkey-flowers (42) and which contrasts to evidence from humans that recombination rates are higher in introns than exons (47). One possibility for the pattern seen in the finches is that DSBs preferentially initiate in the first few exons and their resolution occurs in both nearby exons and introns. The specific mechanism by which DSBs would preferentially initiate in exons is unknown, but the pattern is consistent with an important role for chromatin marks that distinguish exons from introns (45).

\section{Contrasting tempos of broad- and fine scale recombination rate evolution}

Median recombination rates across and within chromosomes vary over nearly six orders of magnitude (Fig. S9, Fig. S18), creating a heterogeneous landscape of broad-scale recombination rates across the genome, with regions of elevated recombination near telomeres and large intervening deserts (as found in zebra finch pedigree data; (30)). This pattern is most pronounced on the sex chromosome $Z$, which has recombination rates that are more than two orders of magnitude lower compared to the chromosome $1 \mathrm{~A}$, the most similarly sized autosome (Fig. S9, (30)). While in both zebra finch and long-tailed finch, cytological data indicate that 
chromosome $Z$ harbors a pericentric inversion polymorphism over most of its length $(48,49)$, such an inversion is unlikely to explain our findings (23).

Between zebra finch and long-tailed finch, broad-scale rates are highly similar, with a genome-wide correlation of 0.82 and 0.84 across $10 \mathrm{~kb}$ and $1 \mathrm{Mb}$ windows, respectively (Fig. 6 , Fig. S18). Despite this broad-scale concordance, we infer that some genomic regions between the two species have very different rates of recombination (Fig. S19). Moreover, at a greater evolutionary distance, broad-scale patterns differ markedly; unlike zebra finch and long-tailed finch, the collared flycatcher ( 19 myr diverged) has a fairly homogeneous recombination landscape (50). This variation in broad-scale rates is particularly notable because, in many species, shifts in broad-scale recombination patterns can be explained almost entirely by chromosomal rearrangements, shifts in karyotypes, and changes in chromosome lengths $(9,51$, 52). However, there is no obvious pattern by which chromosomal rearrangements drive differences in recombination rates between zebra finch and long-tailed finch (Fig. S19), and, despite harboring a number of small inversions between them, collared flycatcher and zebra finch have similar karyotypes and syntenic genomes (50). That broad-scale recombination patterns have changed across the same phylogenetic breadth for which we see hotspot conservation suggests two non-exclusive possibilities: either the heats or locations of some hotspots have evolved, or rates have changed in regions that fall outside of our operational definition of hotspots.

\section{Understanding the impact of recombination on the genome}

Given the marked variation in recombination rates across the genome, we consider the consequences for genome evolution. First, we note that increased recombination rates drive increased GC content in the genome, presumably via gBGC, and we see this phenomenon both at the genome-wide scale (Fig. 3A-B) and the scale of hotspots (Fig. 4). An extreme example is provided by the pseudoautosomal region (PAR), which we identified on an unassembled scaffold from chromosome $Z$ using estimates of coverage in males and females. We confirmed the PAR by inferring homology to PARs identified in medium ground finch and collared flycatcher (Fig. S20). The PAR is short-estimated to be just $450 \mathrm{~kb}$-and is subject to an obligate crossover in every female meiosis (53); as such, it has very high recombination rates. The consequence is visible in the high $\mathrm{GC}^{*}$ for the PAR, which exceeds estimates of $\mathrm{GC}^{*}$ across most of the rest of chromosome $Z$ in both species (Fig. 3C-D).

Further, as has been reported for many other organisms, notably humans and Drosophila $(54,55)$, our results suggest that recombination is positively correlated with levels of nucleotide diversity, particularly on the Z (Fig. S21, S22). This observation is consistent with widespread effects of linked selection in these species (56).

\section{Conclusion}

Finches lack PRDM9 yet nonetheless harbor hotspots, with recombination concentrated at promoter features that likely denote greater accessibility to the cellular recombination machinery. In sharp contrast to apes and mice, the hotspot locations are conserved among species several millions of years diverged and likely over tens of millions of years. These results suggest that the genetic architecture of recombination influences the rate at which hotspots evolve. Whereas the binding specificity of PRDM9 drives rapid turnover, the reliance on 
accessible, functional genomic features leads to stasis. This hypothesis accords with recent results in yeast, in which recombination is concentrated at promoters, and hotspots are stable in intensity and location over tens of millions of years (33). To further investigate how deeply this stasis extends and explore the taxonomic generality of these findings, the approaches illustrated here can be applied to the more than forty bird species with available genomes (19) and beyond. In doing so, we will begin to better understand why species differ so drastically in their specification of hotspots and, in particular, why a subset rely on PRDM9.

\section{References and Notes}

1. B. de Massy, Initiation of meiotic recombination: how and where? Conservation and specificities among eukaryotes. Annu Rev Genet 47, 563-599 (2013).

2. S. Myers et al., Drive against hotspot motifs in primates implicates the PRDM9 gene in meiotic recombination. Science 327, 876-879 (2010).

3. I. L. Berg et al., PRDM9 variation strongly influences recombination hot-spot activity and meiotic instability in humans. Nature genetics 42, 859-863 (2010).

4. A. L. Williams et al., Non-crossover gene conversions show strong GC bias and unexpected clustering in humans. B. de Massy, Ed., (2015).

5. F. Baudat et al., PRDM9 is a major determinant of meiotic recombination hotspots in humans and mice. Science 327, 836-840 (2010).

6. P. L. Oliver et al., Accelerated evolution of the Prdm9 speciation gene across diverse metazoan taxa. PLoS genetics 5, e1000753 (2009).

7. A. G. Hinch et al., The landscape of recombination in African Americans. Nature 476, 170 175 (2011).

8. L. S. Stevison et al., (2015).

9. A. Auton et al., A fine-scale chimpanzee genetic map from population sequencing. Science 336, 193-198 (2012).

10. K. Brick, F. Smagulova, P. Khil, R. D. Camerini-Otero, G. V. Petukhova, Genetic recombination is directed away from functional genomic elements in mice. Nature 485, 642-645 (2012).

11. A. Auton et al., Genetic recombination is targeted towards gene promoter regions in dogs. PLoS genetics 9, e1003984 (2013).

12. J. Pan et al., A hierarchical combination of factors shapes the genome-wide topography of yeast meiotic recombination initiation. Cell 144, 719-731 (2011).

13. K. Choi et al., Arabidopsis meiotic crossover hot spots overlap with H2A.Z nucleosomes at gene promoters. Nature genetics 45, 1327-1336 (2013).

14. A. H. Chan, P. A. Jenkins, Y. S. Song, Genome-wide fine-scale recombination rate variation in Drosophila melanogaster. PLoS genetics 8, e1003090 (2012).

15. C. Smukowski Heil, C. Ellison, M. Dubin, M. Noor, (2015).

16. A. Wallberg, S. Glémin, M. T. Webster, Extreme Recombination Frequencies Shape Genome Variation and Evolution in the Honeybee, Apis mellifera. (2015).

17. T. Kaur, M. V. Rockman, Crossover heterogeneity in the absence of hotspots in Caenorhabditis elegans. Genetics 196, 137-148 (2014). 
18. G. Zhang et al., Comparative genomics reveals insights into avian genome evolution and adaptation. Science 346, 1311-1320 (2014).

19. E. D. Jarvis et al., Whole-genome analyses resolve early branches in the tree of life of modern birds. Science 346, 1320-1331 (2014).

20. J. A. St John et al., Sequencing three crocodilian genomes to illuminate the evolution of archosaurs and amniotes. Genome biology 13, 415 (2012).

21. G. Coop, M. Przeworski, An evolutionary view of human recombination. Nature reviews. Genetics 8, 23-34 (2007).

22. K. Choi, I. R. Henderson, Meiotic recombination hotspots - a comparative view. The Plant journal : for cell and molecular biology, (2015).

23. Materials and methods are available as supplementary materials on Science Online.

24. W. C. Warren et al., The genome of a songbird. Nature 464, 757-762 (2010).

25. G. Watterson, On the number of segregating sites in genetical models without recombination. Theoretical population biology 7, 256-276 (1975).

26. F. Tajima, The effect of change in population size on DNA polymorphism. Genetics 123, 597-601 (1989).

27. E. M. Leffler et al., Revisiting an old riddle: what determines genetic diversity levels within species? PLoS biology 10, e1001388 (2012).

28. O. Delaneau, B. Howie, A. J. Cox, J.-F. Zagury, J. Marchini, Haplotype estimation using sequencing reads. The American Journal of Human Genetics 93, 687-696 (2013).

29. A. L. Williams, D. E. Housman, M. C. Rinard, D. K. Gifford, Rapid haplotype inference for nuclear families. Genome biology 11, R108 (2010).

30. N. Backstrom et al., The recombination landscape of the zebra finch Taeniopygia guttata genome. Genome research 20, 485-495 (2010).

31. P. Fearnhead, SequenceLDhot: detecting recombination hotspots. Bioinformatics 22, 3061-3066 (2006).

32. F. Pratto et al., DNA recombination. Recombination initiation maps of individual human genomes. Science 346, 1256442 (2014).

33. I. K. Lam, Scott. (in review).

34. A. Nicolas, D. Treco, N. P. Schultes, J. W. Szostak, An initiation site for meiotic gene conversion in the yeast Saccharomyces cerevisiae. Nature 338, 35-39 (1989).

35. N. Sueoka, On the genetic basis of variation and heterogeneity of DNA base composition. Proceedings of the National Academy of Sciences of the United States of America 48, 582 (1962).

36. J. Meunier, L. Duret, Recombination drives the evolution of GC-content in the human genome. Molecular biology and evolution 21, 984-990 (2004).

37. B. Nabholz, A. Kunstner, R. Wang, E. D. Jarvis, H. Ellegren, Dynamic evolution of base composition: causes and consequences in avian phylogenomics. Molecular biology and evolution 28, 2197-2210 (2011).

38. C. C. Weber, B. Boussau, J. Romiguier, E. D. Jarvis, H. Ellegren, Evidence for GC-biased gene conversion as a driver of between-lineage differences in avian base composition. Genome biology 15, 549 (2014).

39. F. Smagulova et al., Genome-wide analysis reveals novel molecular features of mouse recombination hotspots. Nature 472, 375-378 (2011). 
40. G. Zhang, P. Parker, B. Li, H. Li, J. Wang, The genome of Darwin's Finch (Geospiza fortis). GigaScience 1, 13 (2012).

41. H. Ellegren et al., The genomic landscape of species divergence in Ficedula flycatchers. Nature 491, 756-760 (2012).

42. U. Hellsten et al., Fine-scale variation in meiotic recombination in Mimulus inferred from population shotgun sequencing. Proceedings of the National Academy of Sciences of the United States of America 110, 19478-19482 (2013).

43. L. Giraut et al., Genome-wide crossover distribution in Arabidopsis thaliana meiosis reveals sex-specific patterns along chromosomes. PLoS genetics 7, e1002354 (2011).

44. A. M. Deaton, A. Bird, CpG islands and the regulation of transcription. Genes \& development 25, 1010-1022 (2011).

45. P. A. Jones, Functions of DNA methylation: islands, start sites, gene bodies and beyond. Nature Reviews Genetics 13, 484-492 (2012).

46. E. Wijnker et al., The genomic landscape of meiotic crossovers and gene conversions in Arabidopsis thaliana. eLife 2, e01426 (2013).

47. A. Kong et al., Fine-scale recombination rate differences between sexes, populations and individuals. Nature 467, 1099-1103 (2010).

48. L. Christidis, Chromosomal evolution within the family Estrildidae (Aves) I. The Poephilae. Genetica 71, 81-97 (1986).

49. Y. Itoh, K. Kampf, C. N. Balakrishnan, A. P. Arnold, Karyotypic polymorphism of the zebra finch Z chromosome. Chromosoma 120, 255-264 (2011).

50. T. Kawakami et al., A high-density linkage map enables a second-generation collared flycatcher genome assembly and reveals the patterns of avian recombination rate variation and chromosomal evolution. Molecular ecology 23, 4035-4058 (2014).

51. B. L. Dumont, B. A. Payseur, Evolution of the genomic rate of recombination in mammals. Evolution 62, 276-294 (2008).

52. M. I. Jensen-Seaman et al., Comparative recombination rates in the rat, mouse, and human genomes. Genome research 14, 528-538 (2004).

53. S. P. Otto et al., About PAR: the distinct evolutionary dynamics of the pseudoautosomal region. Trends in genetics : TIG 27, 358-367 (2011).

54. M. W. Nachman, Single nucleotide polymorphisms and recombination rate in humans. TRENDS in Genetics 17, 481-485 (2001).

55. D. J. Begun, C. F. Aquadro, Levels of naturally occurring DNA polymorphism correlate with recombination rates in D. melanogaster. (1992).

56. B. Charlesworth, M. Morgan, D. Charlesworth, The effect of deleterious mutations on neutral molecular variation. Genetics 134, 1289-1303 (1993).

57. S. C. Griffith, S. R. Pryke, M. Mariette, Use of nest-boxes by the Zebra Finch (Taeniopygia guttata): implications for reproductive success and research. Emu 108, 311-319 (2009).

58. L. A. Rollins, N. Svedin, S. R. Pryke, S. C. Griffith, The role of the Ord Arid Intrusion in the historical and contemporary genetic division of long - tailed finch subspecies in northern Australia. Ecology and evolution 2, 1208-1219 (2012).

59. G. Pesole et al., Structural and functional features of eukaryotic mRNA untranslated regions. Gene 276, 73-81 (2001).

60. J. Nylander, MrAIC. pl. Program distributed by the author 1, (2004). 
61. S. Guindon et al., New algorithms and methods to estimate maximum-likelihood phylogenies: assessing the performance of PhyML 3.0. Systematic biology 59, 307-321 (2010).

62. L. Liu, L. Yu, D. K. Pearl, S. V. Edwards, Estimating species phylogenies using coalescence times among sequences. Systematic Biology 58, 468-477 (2009).

63. R. Bouckaert et al., BEAST 2: a software platform for Bayesian evolutionary analysis. PLoS computational biology 10, e1003537 (2014).

64. R. Agate, B. Scott, B. Haripal, C. Lois, F. Nottebohm, Transgenic songbirds offer an opportunity to develop a genetic model for vocal learning. Proceedings of the National Academy of Sciences 106, 17963-17967 (2009).

65. D. M. Hooper, T. D. Price, Rates of karyotypic evolution in Estrildid finches differ between island and continental clades. Evolution 69, 890-903 (2015).

66. T. D. Price et al., Niche filling slows the diversification of Himalayan songbirds. Nature 509, 222-225 (2014).

67. W. Jetz, G. Thomas, J. Joy, K. Hartmann, A. Mooers, The global diversity of birds in space and time. Nature 491, 444-448 (2012).

68. E. D. Jarvis et al., Whole-genome analyses resolve early branches in the tree of life of modern birds. Science 346, 1320-1331 (2014).

69. R. R. Bouckaert, DensiTree: making sense of sets of phylogenetic trees. Bioinformatics 26, 1372-1373 (2010).

70. J. K. Pickrell, J. K. Pritchard, Inference of population splits and mixtures from genomewide allele frequency data. (2012).

71. D. Reich, K. Thangaraj, N. Patterson, A. L. Price, L. Singh, Reconstructing Indian population history. Nature 461, 489-494 (2009).

72. C. Camacho et al., BLAST+: architecture and applications. BMC bioinformatics 10, 421 (2009).

73. E. Birney, M. Clamp, R. Durbin, GeneWise and genomewise. Genome research 14, 988995 (2004).

74. F. Baudat, Y. Imai, B. de Massy, Meiotic recombination in mammals: localization and regulation. Nature reviews. Genetics 14, 794-806 (2013).

75. W. J. Kent, BLAT-the BLAST-like alignment tool. Genome research 12, 656-664 (2002).

76. A. R. Quinlan, I. M. Hall, BEDTools: a flexible suite of utilities for comparing genomic features. Bioinformatics 26, 841-842 (2010).

77. A. McKenna et al., The Genome Analysis Toolkit: a MapReduce framework for analyzing next-generation DNA sequencing data. Genome research 20, 1297-1303 (2010).

78. Q. Zhou et al., Complex evolutionary trajectories of sex chromosomes across bird taxa. Science 346, 1246338 (2014).

79. L. Smeds et al., Genomic identification and characterization of the pseudoautosomal region in highly differentiated avian sex chromosomes. Nature communications 5, 5448 (2014).

80. R. S. Harris, Improved pairwise alignment of genomic DNA. (ProQuest, 2007).

81. G. Lunter, M. Goodson, Stampy: a statistical algorithm for sensitive and fast mapping of Illumina sequence reads. Genome research 21, 936-939 (2011). 
82. H. Li, Aligning sequence reads, clone sequences and assembly contigs with BWA-MEM. arXiv preprint arXiv:1303.3997, (2013).

83. Z. Iqbal, M. Caccamo, I. Turner, P. Flicek, G. McVean, De novo assembly and genotyping of variants using colored de Bruijn graphs. Nature genetics 44, 226-232 (2012).

84. H. Li et al., The sequence alignment/map format and SAMtools. Bioinformatics 25, 20782079 (2009).

85. G. P. Consortium, A map of human genome variation from population-scale sequencing. Nature 467, 1061-1073 (2010).

86. S. Purcell et al., PLINK: a tool set for whole-genome association and population-based linkage analyses. The American Journal of Human Genetics 81, 559-575 (2007).

87. J. K. Pritchard, M. Przeworski, Linkage disequilibrium in humans: models and data. The American Journal of Human Genetics 69, 1-14 (2001).

88. C. M. Rands et al., Insights into the evolution of Darwin's finches from comparative analysis of the Geospiza magnirostris genome sequence. Bmc Genomics 14, 95 (2013).

89. B. Langmead, S. L. Salzberg, Fast gapped-read alignment with Bowtie 2. Nature methods 9, 357-359 (2012).

90. M. Stephens, P. Donnelly, A comparison of bayesian methods for haplotype reconstruction from population genotype data. The American Journal of Human Genetics 73, 1162-1169 (2003).

91. G. K. Chen, P. Marjoram, J. D. Wall, Fast and flexible simulation of DNA sequence data. Genome research 19, 136-142 (2009).

92. T. L. Bailey et al., MEME SUITE: tools for motif discovery and searching. Nucleic acids research, gkp335 (2009).

93. P. Flicek et al., Ensembl 2012. Nucleic acids research, gkr991 (2011).

94. A. Roberts, L. Pachter, Streaming fragment assignment for real-time analysis of sequencing experiments. Nature methods 10, 71-73 (2013).

95. T. Rausch et al., DELLY: structural variant discovery by integrated paired-end and splitread analysis. Bioinformatics 28, i333-i339 (2012).

\section{Acknowledgements}

This project was started when MP was a Howard Hughes Medical Institute Early Career Scientist and was funded, in part, by Wellcome Trust grants 086786/Z/08/Z to O.V. and 090532/Z/09/Z to the Wellcome Trust Centre for Human Genetics. We thank Bernard de Massy, Corinne Grey, Simon Myers, Trevor Price, Molly Schumer, Jeff Wall, Amy Williams and John Willis for helpful discussions and/or comments on the manuscript; Karene Argoud and Dr. Paolo Piazza at the Genomics Core at the Wellcome Trust Centre for Human Genetics for assistance with lab work; and M. Thomas Gilbert for sharing the zebra finch gene annotations in advance of publication. We are grateful to Isabel Lam and Scott Keeney for sharing their unpublished results and manuscript with us, and for many helpful discussions.

BAM alignment files for genomic data for zebra finch, long-tailed finch, and doublebarred finch are available at XXX. Sequence reads for RNAseq experiments in zebra finch are available at XXX. Filtered variant call files (VCFs) are available for zebra finch, long-tailed finch, and double-barred finch at XXX. Masked genome files for zebra finch, long-tailed finch, and 
double-barred finch and the reconstructed ancestral genome are available at XXX. Raw recombination output from LDhelmet for zebra finch and long-tailed finch is available at XXX. All scripts and an electronic lab notebook for this work are available at https://github.com/singhal/postdoc and https://github.com/singhal/labnotebook, respectively.

\section{Figures}

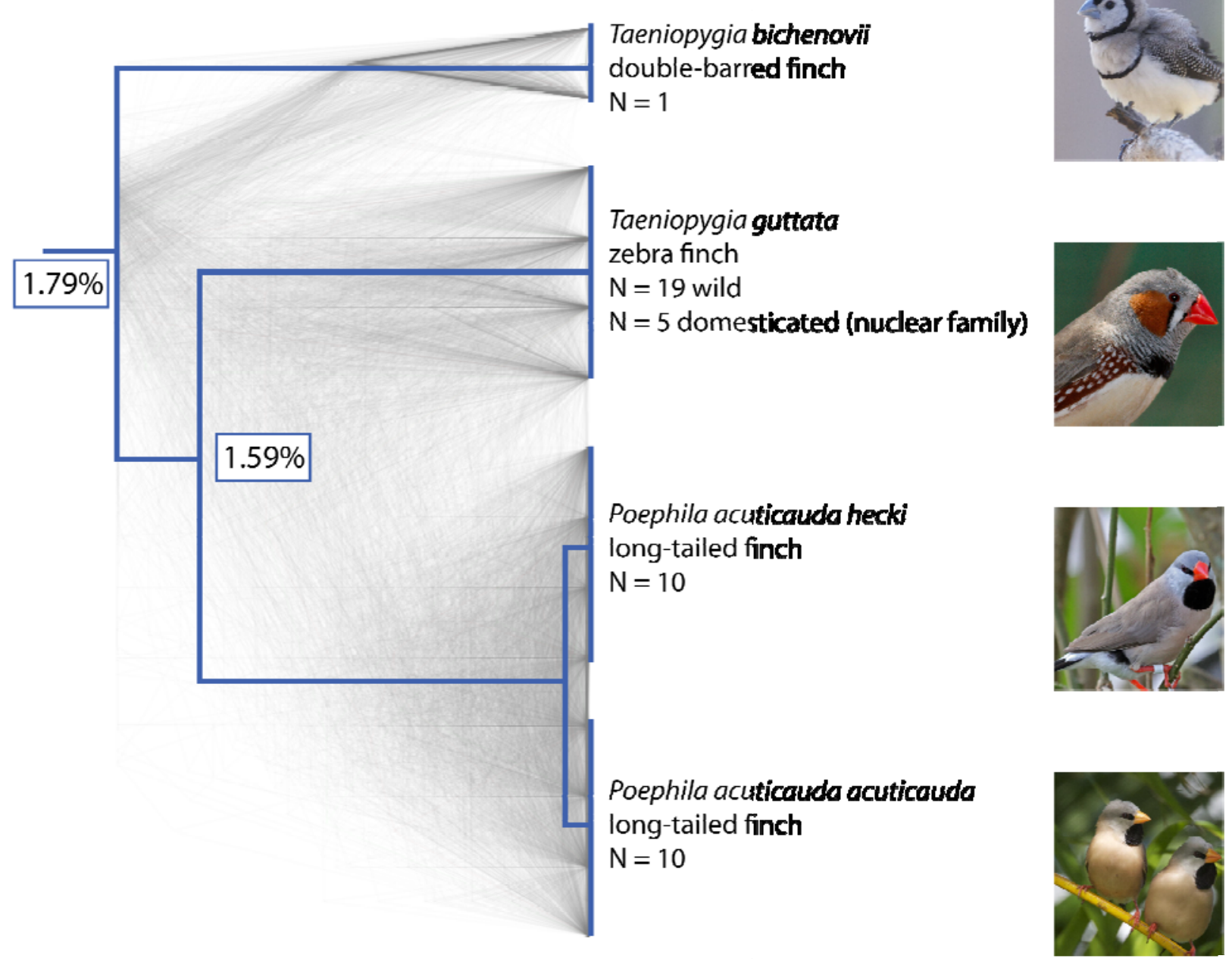

Figure 1: Species tree, gene trees, and sampling numbers for the species in this study: doublebarred finch (Taeniopygia bichenovii), zebra finch (T. guttata), and the two long-tailed finch subspecies (Poephila acuticauda hecki and P. a. acuticauda). Tree rooted with medium ground finch and collared flycatcher (Geospiza fortis and Ficedula albicollis; not shown). Shown in gray are 1000 gene trees, which were used to infer the species tree (23). The pairwise divergence between species is indicated at nodes, as measured by the genome-wide average across autosomes. Images of birds from Wikimedia Commons. 


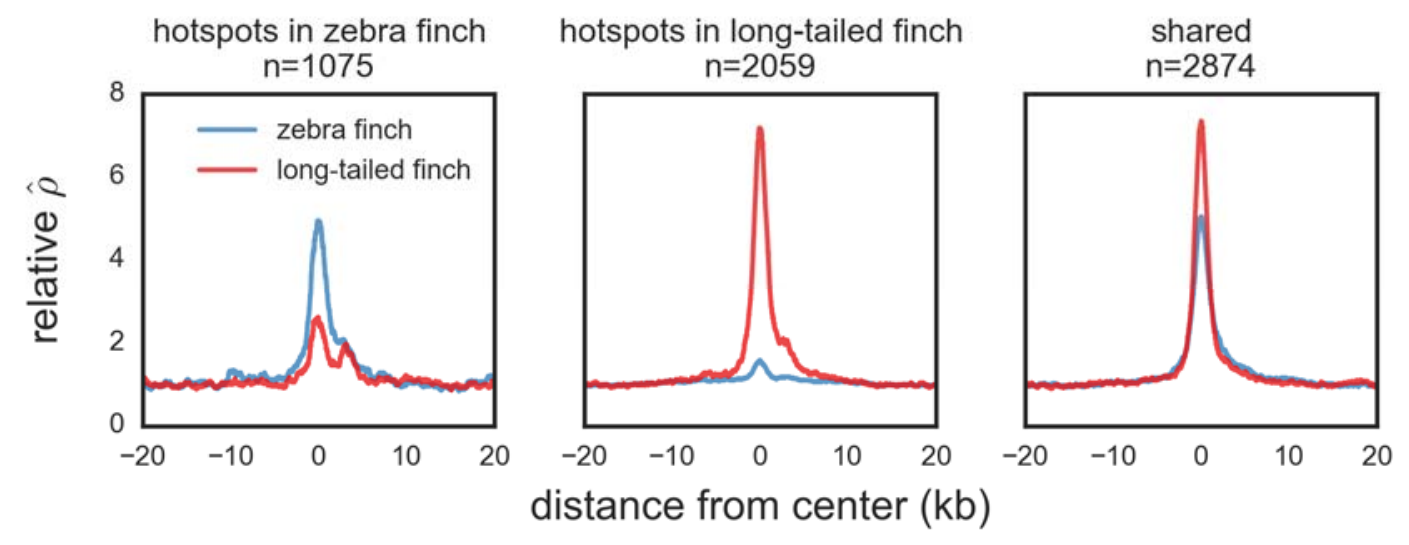

Figure 2: Average relative recombination rate ( ) divided by the background of $20 \mathrm{~kb}$ on either side of the hotspot) across hotspots detected only in zebra finch (Taeniopygia guttata), those detected only in long-tailed finch (Poephila acuticauda), and those inferred as shared in the two species. Shared hotspots are those whose midpoints occur within $3 \mathrm{~kb}$ of each other. The orientation of hotspots is with respect to the genomic sequence and has no functional interpretation.
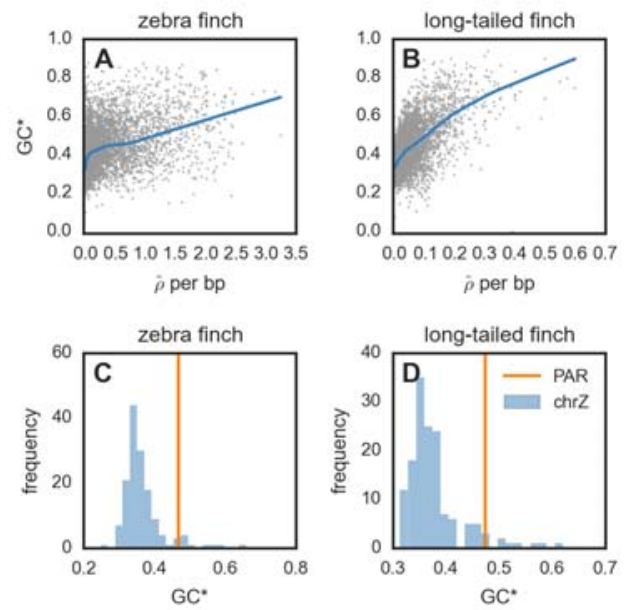

Figure 3: (A-B) Relationship between equilibrium GC content $\left.\left(G C^{*} ; 23\right)\right)$ and for zebra finch (Taeniopygia guttata) and long-tailed finch (Poephila acuticauda) across all autosomal chromosomes. Both $\mathrm{GC}^{*}$ and were calculated across $50 \mathrm{~kb}$ windows with LOESS curves shown for span of 0.2. (C-D) GC* and the pseudoautosomal region (PAR). The histogram shows $\mathrm{GC}^{*}$ for chromosome $Z$ across $500 \mathrm{~kb}$ windows; $\mathrm{GC}^{*}$ for the $450 \mathrm{~kb}$ PAR shown by the vertical line. 


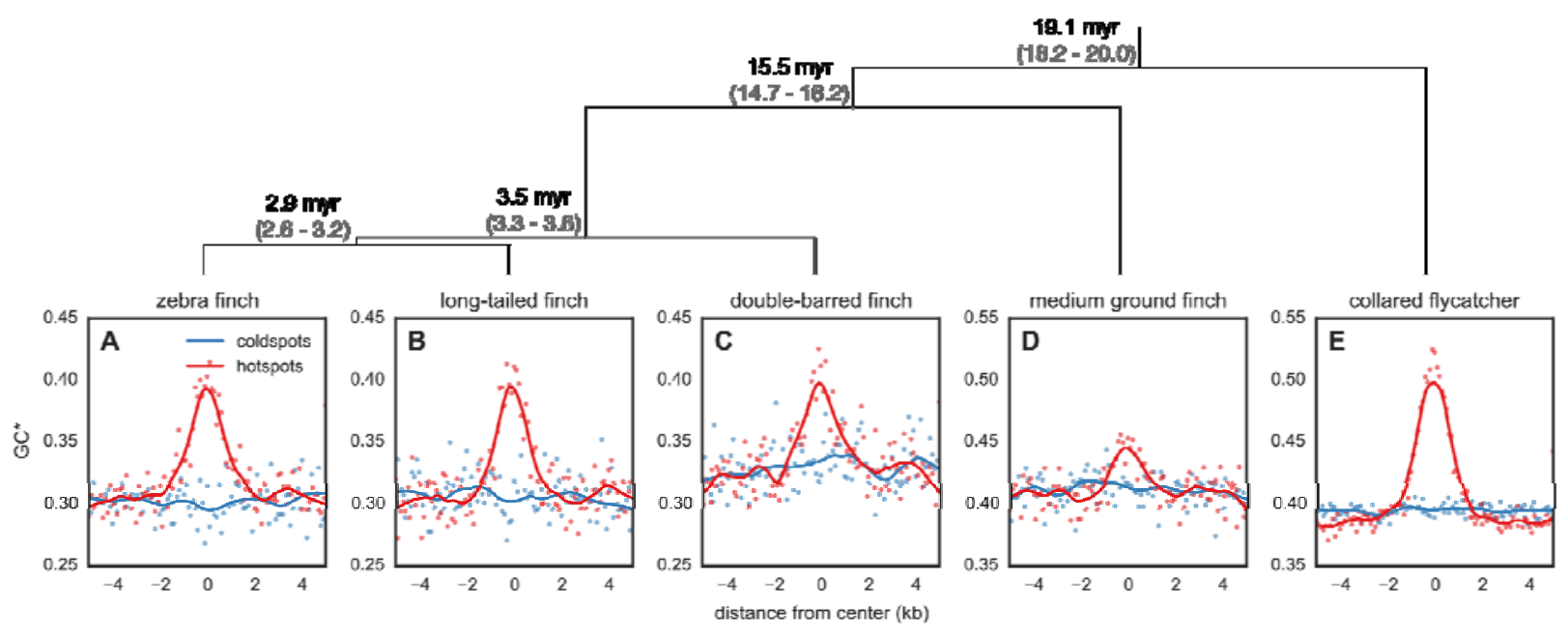

Figure 4: Expected equilibrium GC content $\left(\mathrm{GC}^{\star}\right)$ around hotspots and matched coldspots. Points represent $\mathrm{GC}^{*}$ estimated from the lineage-specific substitutions aggregated in $100 \mathrm{bp}$ bins from the center of all hotspots in (A) zebra finch (Taeniopygia guttata) and (B) long-tailed finch (Poephila acuticauda). GC* for (C) double-barred finch ( T. bichenovii), (D) medium ground finch (Geospiza fortis), and (E) collared flycatcher (Ficedula albicollis) was calculated around hotspots identified as shared between zebra finch and long-tailed finch. LOESS curves are shown for a span of 0.2 . The orientation of hotspots is with respect to the genomic sequence and has no functional intepretation. Species tree (23) shown with estimated divergence times in millions of years (myr) and its 95\% Highest Posterior Density (HPD) in gray. 

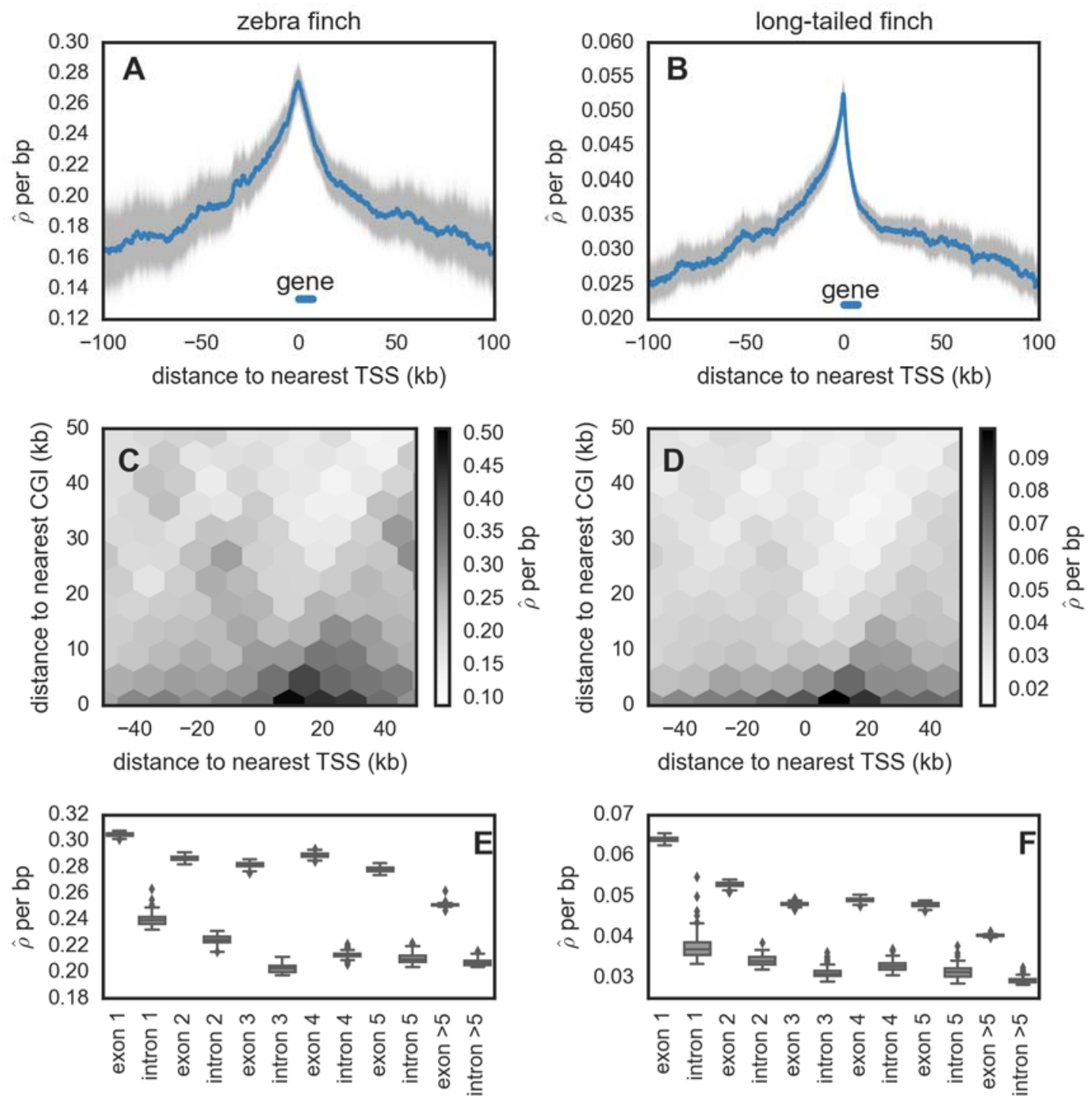

Figure 5: For zebra finch (Taeniopygia guttata) and long-tailed finch (Poephila acuticauda), (AB) estimated recombination rates ( around transcription start sites (TSSs). The distance to TSS was measured accounting for the 5' $\quad 3$ ' orientation of genes. Uncertainty in rate estimates (shown in gray) was estimated by drawing 100 bootstrap samples and recalculating means. The median gene length is indicated. (C-D) shown as a function of both distance to the nearest TSS and distance to the nearest $\mathrm{CpG}$ island (CGI). Data on CGI in zebra finch were downloaded from the UCSC Genome Browser CGI track and were used for long-tailed finch as well. (E-F) within exons and introns for genes that have 5 exons $(n=10,722)$. See Fig. S29 for simulation results that suggest the inference of higher background in exons does not reflect differences in diversity levels between exons and introns. 

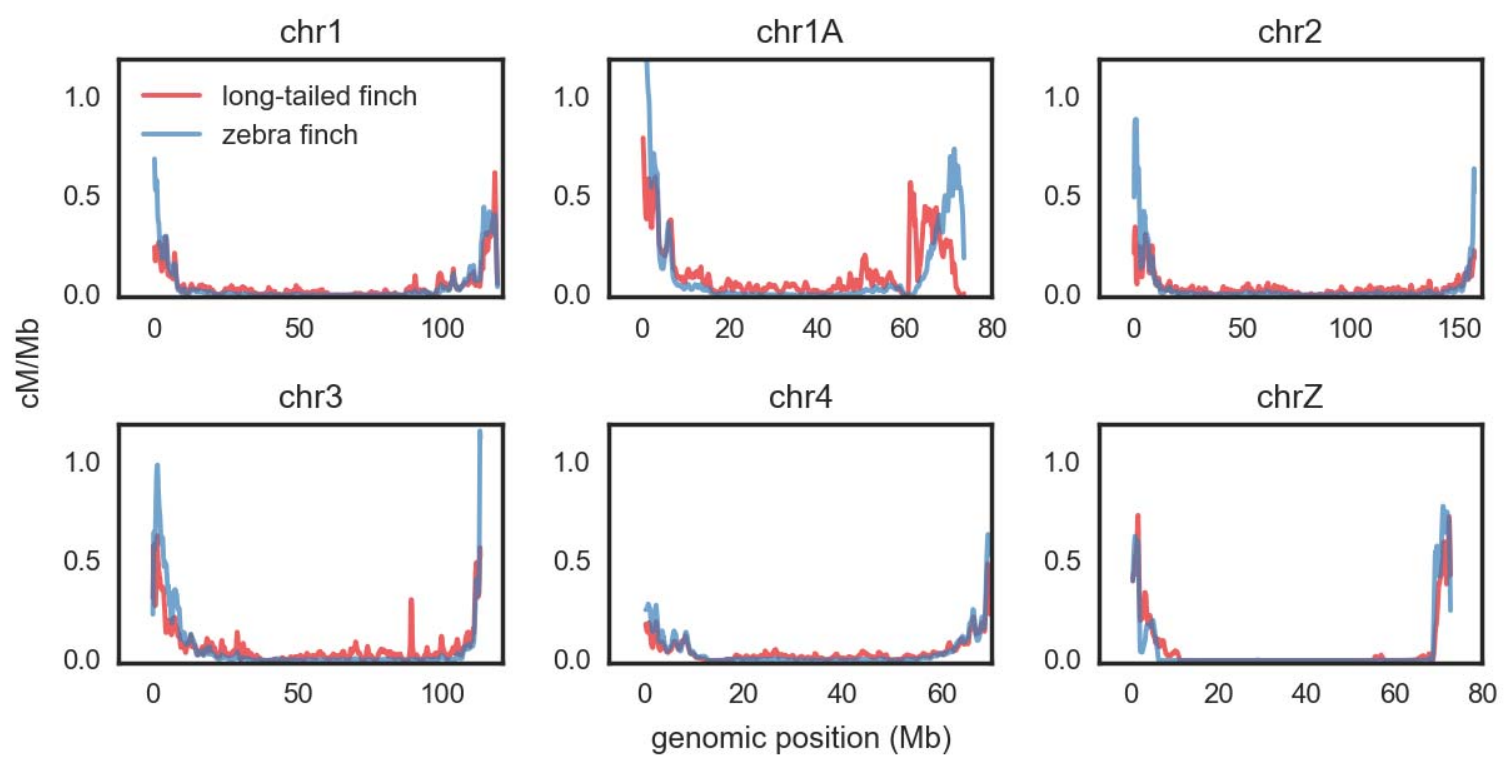

Figure 6: Estimated recombination rate (cM/Mb; obtained from (23)) for zebra finch (Taeniopygia guttata) and long-tailed finch (Poephila acuticauda), shown as rolling means calculated across $100 \mathrm{~kb}$ windows. We show only the five largest autosomal chromosomes and chromosome Z; an expanded version of this figure, showing all chromosomes, is shown in Fig. S18. Rate estimates for chromosome $Z$ should be taken with caution (23).

\section{Supplementary Methods}

Materials and Methods

Figures S1-S29

Tables S1-S6 